\title{
Clusters of galaxies with SPIDERS (The Spectroscopic IDentification of eROSITA Sources)
}

\author{
Nicolas Clerc* \\ IRAP, Université de Toulouse, CNRS, UPS, CNES, Toulouse, France \\ E-mail: nicolas.clerceirap.omp.eu
}

In this presentation I discuss current large surveys of galaxy clusters in the X-ray wavebands, with special emphasis on SPIDERS. It is one of the most ambitious observational programs aimed towards detecting, confirming, cataloguing and interpreting large samples (several thousands) of $\mathrm{X}$-ray detected galaxy clusters, in an effort driven by precision cosmology and galaxy cluster population studies. I introduce some interesting scientific and technical challenges faced in this enterprise and I suggest ways of addressing them by taking examples originating from two widearea, deep, XMM-Newton surveys: XMM-XXL and X-CLASS.

XII Multifrequency Behaviour of High Energy Cosmic Sources Workshop

12-17 June

Palermo, Italy

* Speaker. 


\section{Galaxy cluster cosmology and large $X$-ray surveys}

Wide-area astronomical surveys reveal the large-scale structure in our Universe and support our knowledge of the processes governing its formation and its evolution. Clusters and groups of galaxies form relatively late as a result of the growth of primordial density fluctuations in the expanding Universe. They carry the history of the formation of the cosmic web, comprised of both dark and baryonic matter. The hot baryonic gas and the galaxies falling into such deep gravitational potential wells interact, equilibrate, evolve, enrich each other. Clusters of galaxies allow constraining cosmological models and their number count is a precision probe of dark energy and structure growth altogether. It is complementary of other cosmological probes such as supernovae, baryonic acoustic oscillations, cosmological microwave background (CMB).

\section{Cosmological interpretation of an X-ray cluster survey}

A survey is made of X-ray observations, it also consists in a well-devised methodology to extract relevant cosmological constraints and physics. To illustrate this point, I discuss results from two state-of-the-art wide XMM-Newton surveys.

\subsection{From observations to population statistics: the XMM-XXL 100 brightest clusters}

The XMM-XXL survey It is made of two contiguous fields of size about $25 \mathrm{deg}^{2}$ each, located in two areas of the sky intensively covered by deep and homogeneous multi-wavelength observations [1]. XMM-XXL consists in $10 \mathrm{ks}$ deep overlapping exposures of very sensitive XMMNewton images, for a total of $7 \mathrm{Ms}$ time. This large and medium-deep survey is designed for detecting and characterising 450 galaxy clusters up to $z \sim 1$. The combination of i) a dedicated multi-wavelength follow-up strategy, ii) a controlled selection function and iii) a sensitivity (a few $10^{-14} \mathrm{ergs} \mathrm{s}^{-1} \mathrm{~cm}^{-2}$ ) well-suited to the discovery and characterisation of groups and clusters, enable XMM-XXL to study the nature of dark energy.

Cluster catalogue The publication [2] lists the 100 (X-ray) brightest galaxy clusters together in a series of papers detailing population studies and highlights on individual objects. Spectroscopic observations led to $97 \%$ of these systems being confirmed, they provided precise distances leading to measurements of cluster luminosity, $R_{500}{ }^{1}$ and bulk gas temperature. From these measurements an estimate of the total cluster mass is obtained using a weak-lensing mass-temperature relation calibrated within the survey itself [3]. The XXL-100 clusters populate a new area in the "massredshift" parameter space (black crosses in Fig. 1) at typical masses around few $10^{14} M_{\odot}$ peaking at $z \sim 0.3-0.4$. In this figure, the ROSAT datapoints mostly originate from the $1 \mathrm{RXS}$ version of the survey [4].

Scaling relations Combining optical redshift measurements and X-ray measurements, the luminositytemperature (L-T) relation of the sample reveals non-gravitational physics in groups and clusters. It has been widely studied on numerous samples impacted by - and to a lesser extent, corrected from

\footnotetext{
${ }^{1}$ The cluster-centric radius enclosing an amount of matter whose average density is 500 times the critical density of the Universe at the considered redshift.
} 


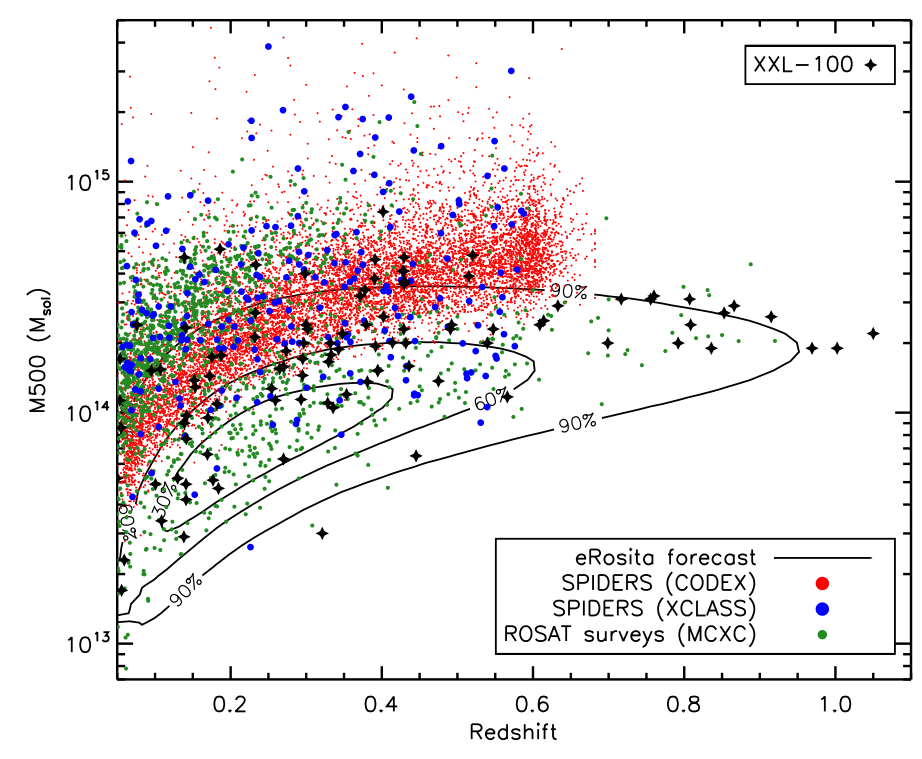

Figure 1: Mass-redshift distribution of the various samples discussed in this paper. Contours highlight the expected location of eROSITA all-sky survey galaxy clusters after four years (maximum depth), assuming a flux limit as in [5].

- a variety of selection biases. The XXL-100 L-T relation [6] takes care of modelling selection biases. This allows to investigate how the L-T evolves in time: it is found that the evolution is consistent with self-similar expectations. Exploiting weak-lensing information from the CFHTLens survey (e.g. [7]) a total mass estimate is compared to their gas temperature for 38/100 systems. Using such a measurement, which is less prone to hydrostatic biases or baryonic physics, shows that the XXL-100 mass-temperature (M-T) relation has a slope steeper than expected from self-similar arguments, while deeper/more observations will likely provide enough leverage to study the M-T evolution with redshift.

Cluster population statistics The luminosity function of clusters is compared to expectations derived from ab initio cosmological modelling, folding the theoretical cluster mass function into the scaling relations derived from survey data and accounting for selection effects. The 100 brightest XXL cluster luminosity function matches well theoretical expectations, no evolution is found with redshift [2]. The redshift histogram of XXL-100 objects is also compatible with theoretical predictions, as long as WMAP-9 cosmological parameters [8] are considered. Switching to PlanckCMB 2015 parameters [9] predicts too many clusters as compared to observations, a result likely due to a higher value of $\sigma_{8}$.

\subsection{A self-consistent approach to cosmological constraints using CR-HR diagrams}

The previous section illustrates a fully self-consistent comparison of theoretical models to $\mathrm{X}$-ray cluster survey data. However in such framework, modifying one or several cosmological parameters requires to recalculate all quantities invoking distances, dimming, etc. This calls for 
performing cosmological studies directly in the space of observables, rather than of computed quantities.

The CR-HR method and its developments In a forecast study [10] we show that projecting a well-selected X-ray cluster population in the "count-rate" (CR or X-ray flux) versus "hardness ratio" (HR or X-ray colour) plane is a powerful method to probe cosmological parameters. It by-passes the need for computing individual cluster masses. Total masses indeed are the fundamental quantities predicted by cosmological models, they are converted into X-ray observables by using parametric scaling relations and instrumental responses. The expected distribution of galaxy clusters in the CR-HR plane responds differently to variations in the different cosmological or scaling relation parameters, thereby enabling to break degeneracies between them (Fig. 2). Adding cluster redshift as an additional parameter ( $z$-CR-HR) provides stronger constraints on evolutiondependent parameters such as $w_{0}$ (dark energy equation of state parameter) or scaling law evolution with redshift. [11] presented an extension of the method by using apparent sizes of the objects as a fourth observable. In all cases, the broad class of CR-HR methods shows interesting as the data is invariant under changes of model parameters. As such, it represents a self-consistent forwardmodelling approach, similarly to e.g. cosmological analyses of the CMB.

CR-HR diagrams in action The methodology of XMM-XXL was applied to the entire XMM archive, by building a serendipitous survey (X-CLASS) of bona-fide extended X-ray sources identified as galaxy clusters. [12] published 421 of these detections, while [13] provided optical followup data (photo-z) for 232 objects up to $z=0.8$. Subsequent updates of the catalogue will bring this number to above 1,000 objects. [12] is a first attempt to apply the CR-HR method to an actual catalogue of galaxy clusters. It presents cosmological constraints on $\Omega_{m}, \sigma_{8}$, and galaxy cluster evolutionary parameters; statistical uncertainties are however large, even with prior information on other parameters, and are expected to be lowered with the inclusion of redshifts [13].

\section{Current developments and near-future prospects with SPIDERS}

This section focuses on very large (full-sky) surveys: SPIDERS, as standalone large program, also appears as a precursor of the eROSITA All-Sky Survey (eRASS).

\subsection{Next steps: the eROSITA all-sky survey}

eROSITA [14] is one of the two high-energy instruments to be mounted on the SRG spacecraft expected to be launched in 2019. Located at the L2 point it will perform 8 scans of the entire sky during 4 years [5]. Equipped with 7 high-throughput X-ray telescopes, eROSITA will be more than the mere successor of ROSAT [15]. Its sensitivity (comparable to XMM-Newton at $1 \mathrm{keV}$ ), angular resolution ( $\sim$ 16.1" on-axis) and spectral resolution (comparable or better than EPIC-pn CCDs onboard XMM) will enable detection of 100,000 galaxy clusters (e.g. [16]) in the soft-band down to $\sim 3-4 \times 10^{-14} \mathrm{ergs} \mathrm{s}^{-1} \mathrm{~cm}^{-2}$ and temperature characterisation of several thousands of them [17]. Roughly speaking, the quality of the eRASS survey will transform the well-studied ROSAT All-sky survey to the depth of an XMM image (Fig. 3). Given the unprecedented amount of sources expected from eRASS, a multi-tiered galaxy cluster follow-up strategy is being devised, involving 

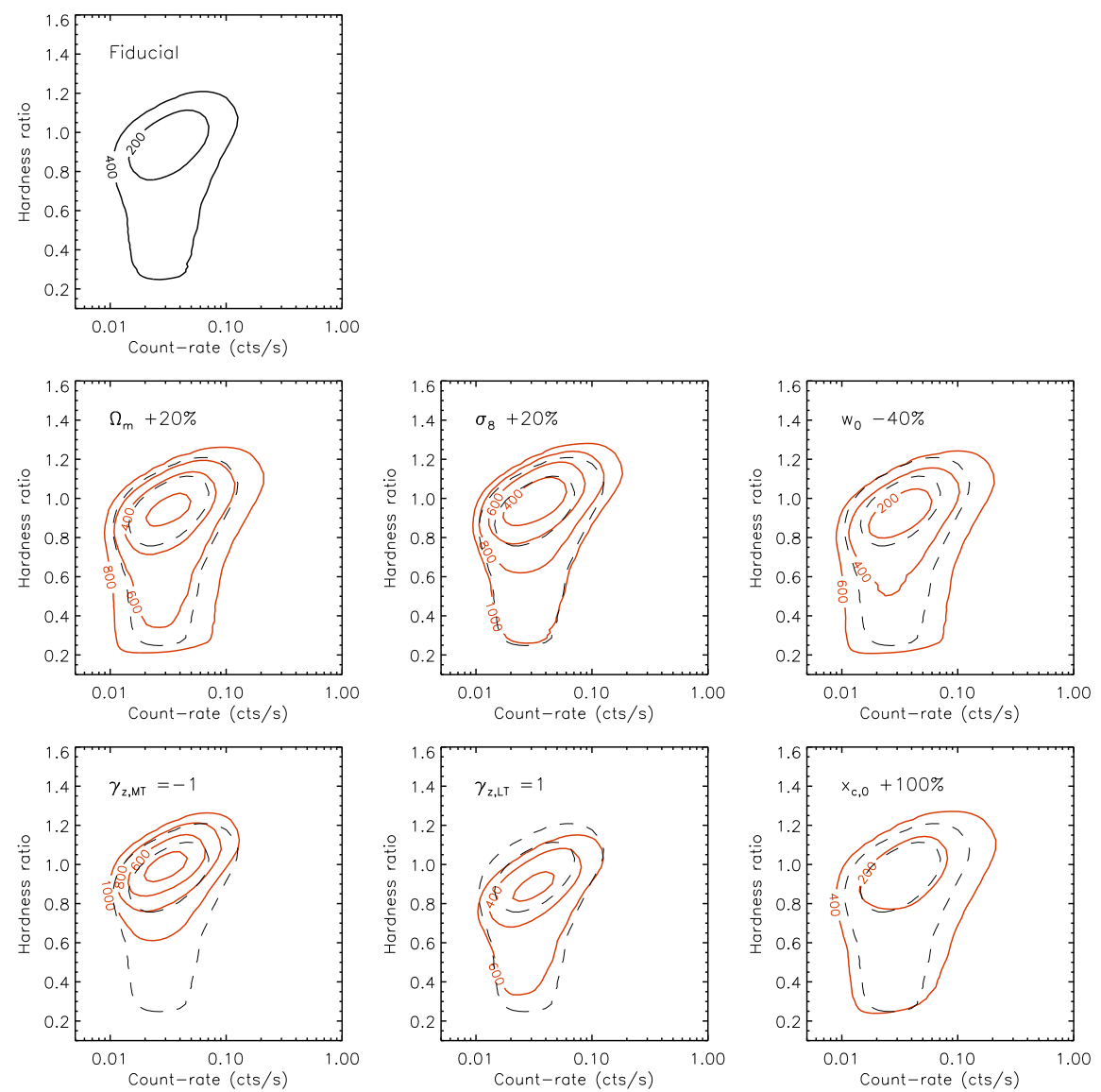

Figure 2: Example CR-HR diagrams and their sensitivity to model parameters (from [10]). Contours enclose predicted distributions of X-ray galaxy clusters for a fiducial model (top-panel and dashed contours in all other panels). The fiducial model is given by $\left\{\Omega_{m}=0.25 ; \sigma_{8}=0.79 ; w_{0}=-1 ; \gamma_{z, \mathrm{LT}}=0 ; \gamma_{z, \mathrm{LT}}=0 ; x_{c, 0}=\right.$ $0.1\}$. Numerics indicate the number of systems within a given contour. Noticeably, contour shape, amplitude and position (plain coloured curves in all six panels) vary differently according to different parameters, whose numerical variation is indicated in legend.

major observing facilities both in the northern and southern hemisphere. In particular, the SDSS-IV project is currently achieving observations in the North through its SPIDERS program.

\subsection{SPIDERS as a follow-up of RASS sources}

Parent samples The observational part of SPIDERS started in 2014 [18] and focuses on faint sources in the ROSAT soft X-ray sky, both AGN [19] and clusters [20]. Indeed, prior to first eRASS catalogues, SPIDERS will secure spectroscopic confirmation of $75 \%$ (about 4,500) CODEX clusters (Finoguenov et al., in prep.) found by filtering RASS faint sources by means of SDSS griz red-sequences [21]. The sample extends to redshift $z \sim 0.6$ with a median mass of $4 \times 10^{14} M_{\odot}$ (red dots in Fig. 1). A small amount of spectroscopic fibres are dedicated to X-CLASS objects (blue dots). In total, SPIDERS will obtain about 10 spectra (and redshifts) per cluster red-sequence over the entire eBOSS area (Fig. 4), hence around 50,000-60,000 galaxy redshifts total. 


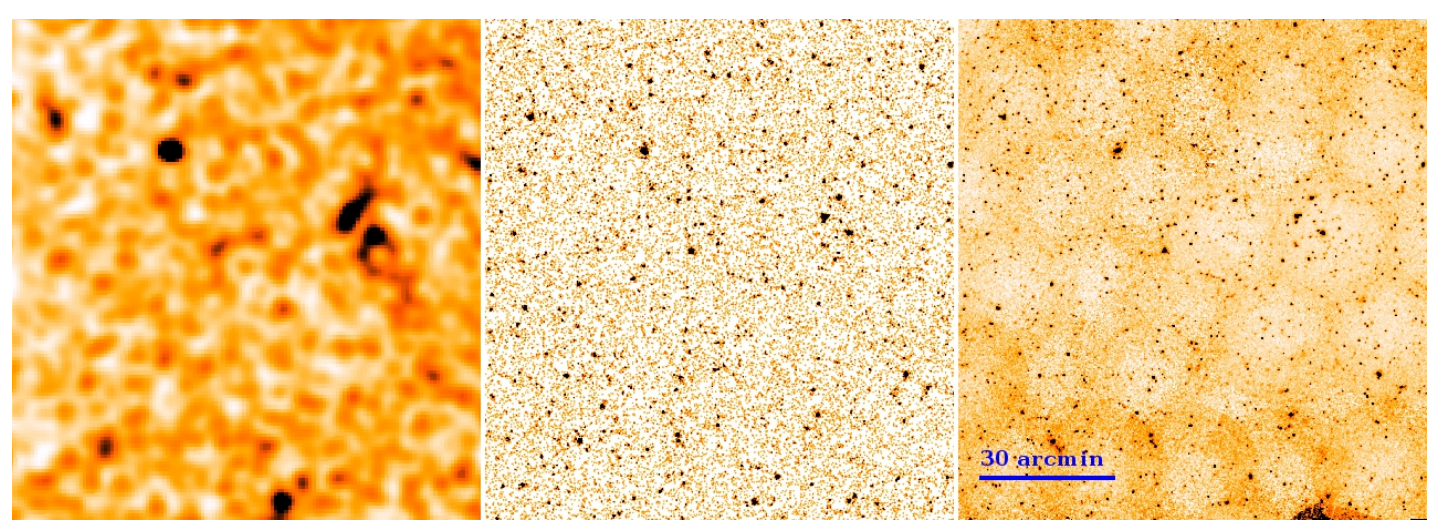

Figure 3: Comparison of an extragalatic field seen in the ROSAT All-sky Survey (left, [0.1-2.4] keV image), eRASS (middle, simulation of the [0.5-2] keV image) and XMM-XXL (right, [0.5-2] keV image). References: [15], Clerc et al. (in prep.) and [1].

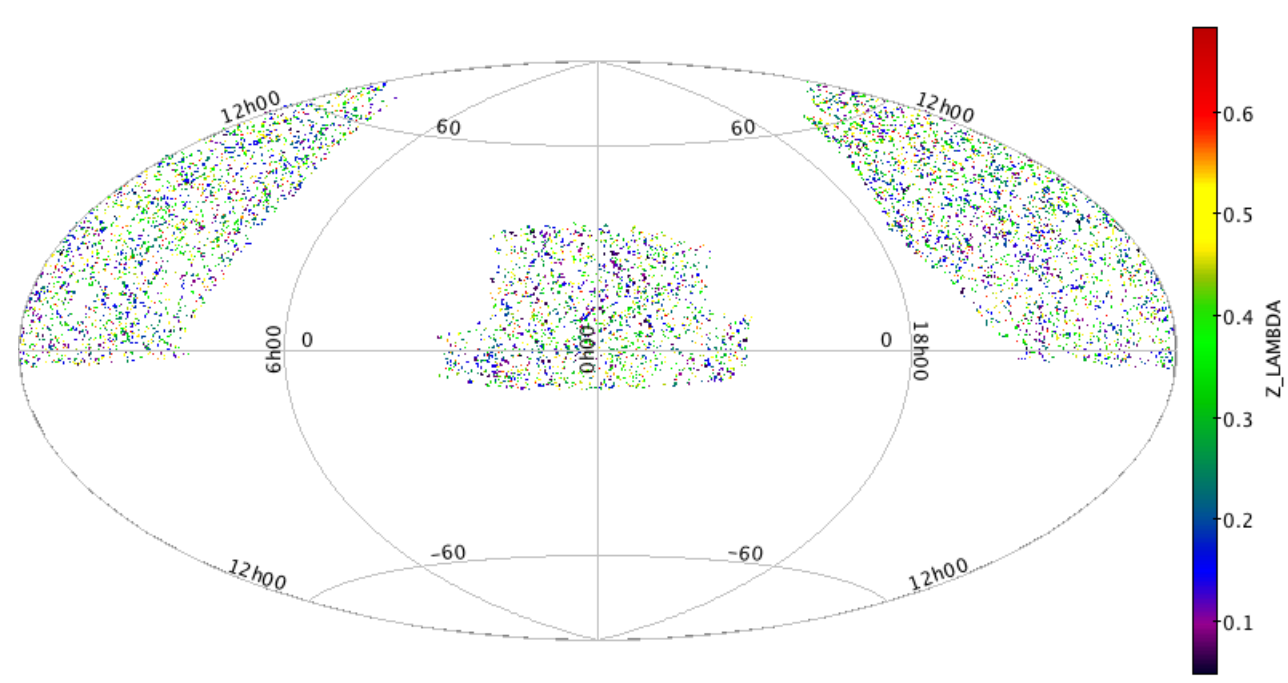

Figure 4: Distribution of all 95,000 spectroscopic targets in the SPIDERS galaxy cluster program (CODEX sample) in equatorial coordinates, colour-coded by the photometric redshift $\left(z_{\lambda}\right)$ of the red-sequence they belong to. Targets are clustered on a $\sim 1-10$ arcmin scale, which represents a challenge for fibre positioning: a priority scheme ensures the most likely cluster members are targeted first.

Demonstration study Using the first $300 \mathrm{deg}^{2}$ of SPIDERS, 230 systems down to low optical richnesses are published as part of a "pilot sample" [22, 20]. Those were confirmed with (at least) three concordant redshifts within their red-sequence, all passed a visual check to confirm the outcome of a standard $\sigma$-clipping algorithm. Averaging the individual galaxy redshifts also provides a systemic redshift compared with the initial photometric redshift $\left(z_{\lambda}\right)$ of the red-sequence (Fig. 5, left). Not only the collected data allows to bring down uncertainties on redshifts by a factor $\sim 10$ compared to photo-z estimates. Also the most densely observed clusters (typically those with more than 15 galaxy redshifts and predominantly located in the low-z part of the survey, Fig. 5, inset) benefit from an estimate of the galaxy velocity dispersion. It correlates well with the X-ray luminosity (Fig. 5, right), as is expected from basic scaling arguments with mass and detailed studies of 
nearby, massive systems (e.g. [23]).
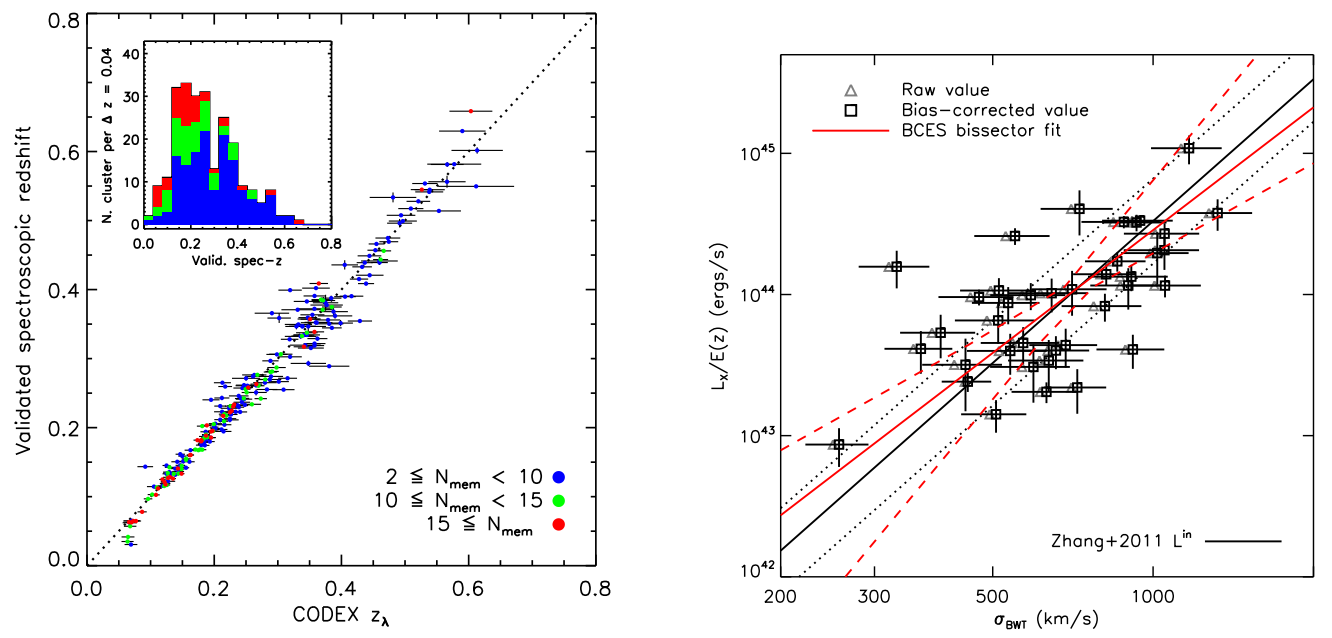

Figure 5: Highlights of the SPIDERS DR13 pilot sample [22, 20]. Left: comparing the photometric redshift $\left(z_{\lambda}\right)$ and spectroscopic redshifts for the 230 confirmed systems, colour-coded by number of members. The inset shows the dsitribution of these of objects as a function of their number of spectroscopic members. Right: comparing the X-ray luminosity and the line-of-sight velocity dispersion for those with more than 15 members.

\section{Conclusions and perspectives}

Shortly after the workshop, SPIDERS delivered a new catalogue of spectroscopically confirmed galaxy clusters with associated galaxy spectra and redshifts as part of the SDSS Data Release 14 [24]. These are rich objects $(\lambda>30)$ with a mean number of redshift per cluster of 13, spread over a 2,500 $\mathrm{deg}^{2}$ sky area. The observations will run until 2020, while catalogs will increase in coverage and depth (lower richnesses). In parallel, identification and studies of the Brightest Cluster Galaxies, refinement of the dynamical masses and tridimensional studies of the clusters are taking place, exploiting the unprecedentedly large dataset offered by SPIDERS.

More broadly this communication attempted to present a perspective on current and upcoming X-ray galaxy cluster surveys with particular emphasis on following challenges:

- Methodology - self-consistent techniques model the X-ray group/cluster population in a cosmological framework, dealing with the broad range of signal-to-noise ratios in the data.

- Statistics - there is a requirement for new approaches to galaxy cluster samples drawn from a pool of $10^{5}$ objects distributed across the entire extragalactic sky.

- Precision - securing accurate redshifts enabling precise positioning of the systems is key to access high-quality mass determinations and faithful mapping of the cosmic web. 


\section{References}

[1] M. Pierre, F. Pacaud, C. Adami, S. Alis, B. Altieri, N. Baran et al., The XXL Survey. I. Scientific motivations - XMM-Newton observing plan - Follow-up observations and simulation programme, A\&A 592 (June, 2016) A1, [1512.04317].

[2] F. Pacaud, N. Clerc, P. A. Giles, C. Adami, T. Sadibekova, M. Pierre et al., The XXL Survey. II. The bright cluster sample: catalogue and luminosity function, A\&A 592 (June, 2016) A2, [1512.04264].

[3] M. Lieu, G. P. Smith, P. A. Giles, F. Ziparo, B. J. Maughan, J. Démoclès et al., The XXL Survey . IV. Mass-temperature relation of the bright cluster sample, A\&A 592 (June, 2016) A4, [1512.03857].

[4] W. Voges, B. Aschenbach, T. Boller, H. Bräuninger, U. Briel, W. Burkert et al., The ROSAT all-sky survey bright source catalogue, A\&A 349 (Sept., 1999) 389-405, [astro-ph/9909315].

[5] A. Merloni, P. Predehl, W. Becker, H. Böhringer, T. Boller, H. Brunner et al., eROSITA Science Book: Mapping the Structure of the Energetic Universe, ArXiv e-prints (Sept., 2012), [1209.3114].

[6] P. A. Giles, B. J. Maughan, F. Pacaud, M. Lieu, N. Clerc, M. Pierre et al., The XXL Survey. III. Luminosity-temperature relation of the bright cluster sample, A\&A 592 (June, 2016) A3, [1512.03833].

[7] T. Erben, H. Hildebrandt, L. Miller, L. van Waerbeke, C. Heymans, H. Hoekstra et al., CFHTLenS: the Canada-France-Hawaii Telescope Lensing Survey - imaging data and catalogue products, MNRAS 433 (Aug., 2013) 2545-2563, [1210 . 8156].

[8] G. Hinshaw, D. Larson, E. Komatsu, D. N. Spergel, C. L. Bennett, J. Dunkley et al., Nine-year Wilkinson Microwave Anisotropy Probe (WMAP) Observations: Cosmological Parameter Results, ApJS 208 (Oct., 2013) 19, [1212.5226].

[9] Planck Collaboration, P. A. R. Ade, N. Aghanim, M. Arnaud, M. Ashdown, J. Aumont et al., Planck 2015 results. XIII. Cosmological parameters, A\&A 594 (Sept., 2016) A13, [1502.01589].

[10] N. Clerc, M. Pierre, F. Pacaud and T. Sadibekova, The cosmological analysis of X-ray cluster surveys - I. A new method for interpreting number counts, MNRAS 423 (July, 2012) 3545-3560, [1109.4440].

[11] M. Pierre, A. Valotti, L. Faccioli, N. Clerc, R. Gastaud, E. Koulouridis et al., The cosmological analysis of X-ray cluster surveys; III. 4D X-ray observable diagrams, ArXiv e-prints (Sept., 2016), [1609.07762].

[12] N. Clerc, T. Sadibekova, M. Pierre, F. Pacaud, J.-P. Le Fèvre, C. Adami et al., The cosmological analysis of X-ray cluster surveys - II. Application of the CR-HR method to the XMM archive, MNRAS 423 (July, 2012) 3561-3583, [1109.4441].

[13] J. Ridl, N. Clerc, T. Sadibekova, L. Faccioli, F. Pacaud, J. Greiner et al., Cosmology with XMM galaxy clusters: the X-CLASS/GROND catalogue and photometric redshifts, MNRAS 468 (June, 2017) 662-684, [1702.04314].

[14] P. Predehl, eROSITA on SRG, Astronomische Nachrichten 338 (Mar., 2017) 159-164.

[15] J. Truemper, ROSAT - A new look at the X-ray sky, Science 260 (June, 1993) 1769-1771.

[16] A. Pillepich, C. Porciani and T. H. Reiprich, The X-ray cluster survey with eRosita: forecasts for cosmology, cluster physics and primordial non-Gaussianity, MNRAS 422 (May, 2012) 44-69, [1111.6587]. 
[17] K. Borm, T. H. Reiprich, I. Mohammed and L. Lovisari, Constraining galaxy cluster temperatures and redshifts with eROSITA survey data, A\&A 567 (July, 2014) A65, [1404.5312].

[18] M. R. Blanton, M. A. Bershady, B. Abolfathi, F. D. Albareti, C. Allende Prieto, A. Almeida et al., Sloan Digital Sky Survey IV: Mapping the Milky Way, Nearby Galaxies, and the Distant Universe, AJ 154 (July, 2017) 28, [1703.00052].

[19] T. Dwelly, M. Salvato, A. Merloni, M. Brusa, J. Buchner, S. F. Anderson et al., SPIDERS: selection of spectroscopic targets using AGN candidates detected in all-sky X-ray surveys, MNRAS 469 (July, 2017) 1065-1095, [1704.01796].

[20] N. Clerc, A. Merloni, Y.-Y. Zhang, A. Finoguenov, T. Dwelly, K. Nandra et al., SPIDERS: the spectroscopic follow-up of X-ray selected clusters of galaxies in SDSS-IV, MNRAS 463 (Dec., 2016) 4490-4515, [1608.08963].

[21] E. S. Rykoff, E. Rozo, M. T. Busha, C. E. Cunha, A. Finoguenov, A. Evrard et al., redMaPPer. I. Algorithm and SDSS DR8 Catalog, ApJ 785 (Apr., 2014) 104, [1303. 3562].

[22] SDSS Collaboration, F. D. Albareti, C. Allende Prieto, A. Almeida, F. Anders, S. Anderson et al., The Thirteenth Data Release of the Sloan Digital Sky Survey: First Spectroscopic Data from the SDSS-IV Survey MApping Nearby Galaxies at Apache Point Observatory, ArXiv e-prints (Aug., 2016), [1608.02013].

[23] Y.-Y. Zhang, T. H. Reiprich, P. Schneider, N. Clerc, A. Merloni, A. Schwope et al., HIFLUGCS: $X$-ray luminosity-dynamical mass relation and its implications for mass calibrations with the SPIDERS and 4MOST surveys, A\&A 599 (Mar., 2017) A138.

[24] B. Abolfathi, D. S. Aguado, G. Aguilar, C. Allende Prieto, A. Almeida, T. Tasnim Ananna et al., The Fourteenth Data Release of the Sloan Digital Sky Survey: First Spectroscopic Data from the extended Baryon Oscillation Sky Survey and from the second phase of the Apache Point Observatory Galactic Evolution Experiment, ArXiv e-prints (July, 2017), [1707.09322].

\section{DISCUSSION}

MARIA PRUZHINSKAYA: How much galaxies (members of the cluster) with spectroscopy are used to determine the cluster redshift? How much clusters with spectroscopically defined redshift are you expected to have in SPIDERS?

NICOLAS CLERC: In the pilot sample presented here, there are in average 8 galaxies per cluster red-sequence spectroscopically identified as members of a SPIDERS galaxy cluster (see Fig. 5, inset) and a robust mean of their individual redshift measurements serves as an estimate of the cluster systemic redshift. The current expectation, depending on the exact richness and completeness cuts, is around 4,500 - 5,000 X-ray clusters confirmed with SPIDERS spectroscopic observations. 